\title{
Association of rs182429 variant in TAGAP with rheumatoid arthritis in Pakistani population
}

\author{
Maria Arshad \\ National University of Sciences \& Technology, H-12, Islamabad, Pakistan, atta-ur-rahman school of \\ applied biosciences Email: maria.arshad14@gmail.com
}

Attya Bhatti

National University of Sciences \& Technology, H-12, Islamabad, Pakistan, atta-ur-rahman school of applied biosciences Email: attyabhatti@gmail.com

Peter John

National University of Sciences \& Technology, H-12, Islamabad, Pakistan, atta-ur-rahman school of applied biosciences Email: pjohn72@hotmail.com

Fazal Jalil

Abdul Wali Khan University Mardan, Mardan, Pakistan, department of biotechnology Email: fazaljalil@awkum.edu.pk

Richard O Williams

University of Oxford, Roosevelt Drive, Oxford OX3 7FY, United Kingdom, kennedy institute of rheumatology Email: richard.williams@kennedy.ox.ac.uk

\section{Corresponding Author}

Dr. Attya Bhatti

Assistant Professor

HoD Healthcare Biotechnology

Atta-ur-Rahman School of Applied Biosciences (ASAB)

National University of Sciences and Technology (NUST)

H-12, Islamabad, Pakistan

Tel: 00925190856128

Email Address: attyabhatti@gmail.com 


\section{Abstract}

Variations in T-cell activation Rho-GTPase activating protein (TAGAP) have been associated with various autoimmune diseases including Rheumatoid Arthritis (RA). The gene is involved in $\mathrm{T}$ cell activation and is therefore of particular interest in context of $\mathrm{T}$ cell-driven autoimmune diseases. Genome-wide association studies suggest association between TAGAP and RA in various populations. This study sought to investigate any association of TAGAP with RA in Pakistani population. For this, two variants in TAGAP ( $r s 182429 \mathrm{~A} / \mathrm{G}$ and $r s 212389 \mathrm{~A} / \mathrm{G})$ were investigated because of their high implication in RA liability of European patients. The variants were genotyped in 186 Pakistani RA patients and 185 controls. No significant difference was observed in distribution of $r s 212389$ between patients and controls $(\mathrm{P}=0.87$ for genotypic frequency and $\mathrm{P}=1.000$ for allelic frequency) which differs from findings in European subjects. Conversely, statistically significant difference was observed in $r s 182429(\mathrm{P}=0.0026$ for genotypic frequency and $\mathrm{P}=0.0373$ for allelic frequency). Heterozygous genotype $\mathrm{AG}$ and allele A were found to be disease susceptible genotype and allele respectively, because of their higher frequency in patients. While higher frequency of genotype GG and allele $\mathrm{G}$ in healthy controls suggests them as protective genotype and allele, respectively, in Pakistani population. This is the first TAGAP association study with RA in Pakistani cohort and its results suggest that the distribution of alleles regarding contribution to RA is actually population specific. In future, novel therapeutic targets for RA can be identified by exploring functional significance of these SNPs.

\section{Keywords}

Rheumatoid arthritis; disease susceptibility; single nucleotide polymorphism; genotyping; case/control study 


\section{Introduction}

Rheumatoid arthritis (RA) is an autoimmune disease characterized by chronic inflammation in the synovial membranes of joints that leads to bone damage and loss of joint function (Firestein 2003). RA affects around $0.5-1 \%$ of the adult population in developed regions (Moreland 2005; McInnes and Schett 2011; Turesson et al. 1999). Its prevalence in Southern Pakistan is reported to be $0.142 \%$ (Hameed et al. 1995) whereas in Northern Pakistan the estimated prevalence is $0.55 \%$ (Farooqi and Gibson 1998). Although the cause of RA is still not clearly known, it is estimated that $60 \%$ of the incidence of the disease is attributed to heritable factors (Chang et al. 2008), with the remainder being attributed to environmental factors (Gibofsky et al. 1978).

Genetic association studies have identified the human leukocyte antigen locus DRB1 (HLADRB1) (Gregersen et al. 1987; Gonzalez-Gay et al. 2002; Moreno et al. 1996; Turesson et al. $2005)$ as the main genetic risk factor for RA. In addition to this region, genome wide association studies (GWAS) have also identified various susceptibility variants and loci associated with incidence of RA in a number of populations. These include PTPN22, STAT4, CTLA4, CD28, CCR6 and many more (Hinks et al. 2005; Kobayashi et al. 2008; Kochi et al. 2010; Liu et al. 1996; Lei et al. 2005; Prasad et al. 2012). The gene encoding T-cell activation Rho GTPase Activating Protein (TAGAP) is one such gene that is located on chromosome 6q25. TAGAP is thought to be involved in the activation of T-cells and is therefore of particular interest in the context of autoimmune disease. Furthermore, risk loci at the TAGAP locus have been identified for various autoimmune diseases like type 1 diabetes, celiac disease, crohn's disease and RA (Connelly et al. 2012; Eyre et al. 2010; Franke et al. 2010; Festen et al. 2011). To date, three SNPs in the TAGAP locus have been associated with susceptibility to RA in European population: $r s 182429$, rs394581 and $r s 212389$ (Chen et al. 2011; Eyre et al. 2010; Stahl et al. 2010; Raychaudhuri et al. 2009). Previously, rs394581 was associated with RA but Eyre et al. 2010 suggested that the association of TAGAP with RA comes mainly from rs 182429. Through conditional logistic regression analysis and LD testing it was suggested that this association may have arisen due to LD between these SNPs (Eyre et al. 2010). On the other hand, the $r s 212389$ polymorphism at the TAGAP locus was recently confirmed as a strong risk allele for RA (Chatzikyriakidou et al. 2013). To determine the association between these risk loci of TAGAP 
and Pakistani RA cohort, we genotyped TAGAP polymorphisms rs212389 and rs 182429, in unrelated RA patients and their matching controls.

\section{Materials and methods}

\subsection{Study subjects}

A total of 371 individuals including 186 RA patients and 185 healthy donors were enrolled in this study. Blood samples and relevant demographic information were obtained from subjects recruited from various rheumatology centers (Military Hospital, Fauji Foundation Hospital Rawalpindi and Rehmat Noor Clinic, Rawalpindi, Pakistan). The clinical diagnosis was made by a certified rheumatologist and patients satisfying American College of Rheumatology (ACR) 1987 criteria (Arnett et al. 1988) were included in the study. Healthy age and gender-matched individuals were also recruited from rheumatologists. Written informed consent was obtained from all individuals before enrolling them in this study. The study was approved by the National University of Sciences and Technology, Pakistan.

Blood samples were collected in $10 \mathrm{ml}$ ethylenediaminetetraacetic acid tubes (BD vacutainer K2 EDTA) and stored at $4^{\circ} \mathrm{C}$ before processing for DNA extraction. Depending upon the blood volume, genomic DNA was extracted using either the standard organic (Phenol/Chloroform) method or the Fermentas Life Sciences DNA purification kit, according to the manufacturer's protocol. The DNA was then quantified by using the NanoDrop ND-1000 Spectrophotometer (Labtech International, UK) and working dilutions of $5 \mathrm{ng} / \mu 1$ were prepared for Taqman assays.

\subsection{Genotyping}

Two TAGAP polymorphisms; rs212389 (assay ID: C_2966116_10) and rs182429 (assay ID: C_563829_10) were genotyped using the TaqMan SNP genotyping assays (Applied Biosystems). For this, the wet method was used in which $2.25 \mu 1$ of all DNA samples were spotted onto a MicroAmp Optical 384-well reaction plate (Applied Biosystems) using a Biohit eLINE electronic pipettor (Sartorius). The reaction mixture was then added according to the manufacturer's protocol. The plate was covered with MicroAmp Optical adhesive film (Applied Biosystems). All samples were amplified in duplicates to assess any error rate and the undermine genotypes were repeated for conformation. Cycling conditions for TaqMan reactions were

followed according to Applied BioSystems TaqMan protocols. PCR amplification and the 
endpoint fluorescence reading were performed using ViiA ${ }^{\mathrm{TM}}$ Real-time PCR system (Applied Biosystems).

\subsection{Statistical analysis}

The allele and genotype frequencies were calculated using the allele counting method and the polymorphisms were tested for deviation from Hardy-Weinberg equilibrium (HWE). Association analysis of the SNPs and difference in the allele and genotype distribution between patients and controls was performed through chi- squared $\left(\chi^{2}\right)$ statistics, two-tailed Fisher's exact test and Z-test by using Graphpad Prism $5 \mathrm{~V}$ software (GraphPad Software, San Diego, CA, USA). Odds Ratio (OR) with $95 \%$ confidence intervals (CI) was also calculated using t-test. The $\mathrm{P}$ value $\leq 0.05$ was considered statistically significant.

\section{Results}

The demographic characteristics of RA patients and controls are shown in Table 1. The mean age at onset of disease was $43 \pm 12$ years with $76 \%$ females, whereas the mean age of healthy controls was $40 \pm 11$ years with $36 \%$ females. Of all RA patients, 95\% were rheumatoid factor (RF) positive and 92\% were anti-cyclic citrullinated peptide (ACCP) positive. The genotype and allele frequency analysis for both variants revealed that for variant $r s 182429(\mathrm{~A} / \mathrm{G})$, the frequency of homozygous AA genotype was 72 (38.7\%) in cases and 71 (38.4\%) in controls; while the frequency of heterozygous AG genotype was 91 (48.9\%) in cases and $67(36.2 \%)$ in controls. The homozygous GG genotype frequency was significantly lower in patients $(23 ; 12.4$ \%) compared to controls $(47 ; 25.4 \%)(p<0.0026)$ (Table2, Fig.1a). In contrast, for variant $r s 212389(\mathrm{~A} / \mathrm{G})$, the frequency of the homozygous AA genotype was $93(49.7 \%)$ in cases and $90(48.7 \%)$ in controls; while the frequency of heterozygous AG genotype was $79(42.7 \%)$ in cases and in controls it was 83 (44.9\%). The homozygous GG genotype frequency was 14 (7.6 $\%)$ and $12(6.5 \%)$ in cases and controls, respectively ( $p=0.87)$. (Table 2, Fig.1b).

From the allele frequency analysis of rs 182429, we found that the A allele frequency was significantly higher in patients (OR $1.32(0.98-1.77) ; p<0.0373)$ compared to the control group (Table 3, Fig.2a). While in rs212389 analysis, the frequency of both A and G alleles were found to be similar in cases and controls (OR 1.00 (0.73-1.374); p=1.00) (Table 3, Fig.2b). The Z-test was also performed to calculate the genotypic and allelic frequency distributions of both SNPs and the results are depicted in Table 4. 


\section{Discussion}

Recent advances in molecular genetics have led to the identification of multiple risk loci and genetic variations in both monogenic and common complex diseases including RA. However, due to their complex etiology, multifactorial traits bear ethnicity specific variations in genetic predisposition, and also share most of the susceptible loci. A large number of genome wide association studies and candidate gene approaches has identified a number of risk loci and variants and have explored their role as RA susceptibility loci (Honne et al. 2016; Jalil et al. 2013; Julià et al. 2016; Stahl et al. 2010). The TAGAP gene has been identified as potentially an important risk locus for RA in a number of populations. Previously, a study in a Pakistani population tested the association of 33 SNPs from 31 loci and reported an association of 12 genes including TAGAP ( $r s 394581 ; p=0.015)$ (Jalil et al. 2013). The TAGAP gene, located on 6q25.3, encodes a member of Rho-GTPase protein family which releases GTP from GTP-bound Rho hence acts as a molecular switch. The TAGAP gene is transiently expressed in activated Tcells and is thought to be important in modulating cytoskeletal changes (Connelly et al. 2014). Little is known about its exact role in immune system but it has been found to be co-expressed with IL-2 and is predicted to play a role in T-cell activation (Mao et al. 2004). Given the importance of T-cells in the pathophysiology of RA, any polymorphism in the TAGAP gene might contribute to the pathogenesis of the disease. This gene has also been associated with other autoimmune diseases like celiac disease, crohn's disease and type 1 diabetes (Eyre et al. 2010; Franke et al. 2010; Festen et al. 2011) with a potentially protective role for anal sepsis in ileocolic crohn's disease (Connelly et al. 2012).

The occurrence of three TAGAP SNPs (rs182429, rs212389 and $r$ s394581) in RA has been well documented by different studies in European populations (Eyre et al. 2010; Raychaudhuri et al. 2009; Stahl et al. 2010). However, out of these three important variants only rs 394581 was previously tested for its association with RA in Pakistani population (Jalil et al. 2013). Therefore, we tested the association of the other two variants with RA in Pakistani patients. The HWE was used to test if the real frequencies deviate from the expected. The analysis revealed that the homozygous AA genotype of variant $r s 182429$ has almost equal frequencies in both cases and controls, while the heterozygous AG genotype has significantly higher frequency in patients than in controls which suggests that the AG genotype might contribute to RA in Pakistani patients. In 
contrast, the homozygous GG genotype had a higher frequency in controls which suggests it has a protective role in RA. Moreover, it was also observed that the A allele is a minor allele which has significantly higher frequency in RA patients. The analysis of the variant $r s 212389$ showed similar genotypic (AA, AG and GG) and allelic frequencies in both cases and controls and is thus suggested to have no association with RA in Pakistan. On the basis of these findings, it is suggested that A allele might be a disease susceptibility allele, while the $G$ allele may have protective role for RA in the Pakistani population. It can be concluded that individuals with the AG genotype for the $r s 182429$ polymorphism are at higher risk of developing RA compared to those with the GG genotype. On the other hand, the rs 212389 polymorphism is not associated with incidence of RA in this Pakistani population which contradicts the findings from the studies which showed that the rs212389 polymorphism is the most important SNP at the TAGAP locus for determining susceptibility to RA (Chen et al. 2011; Chatzikyriakidou et al. 2013).

Besides RA, the presence of TAGAP polymorphisms has also been documented in other autoimmune and inflammatory diseases. A meta-analysis of SNPs from genome wide datasets of crohn's disease (3230 cases, 4829 controls) and celiac disease (768 cases, 1422 controls) has shown that TAGAP locus is a shared risk factor for both these diseases (Festen et al. 2011). Another genome wide meta-analysis study reported a suggestive association of TAGAP locus with multiple sclerosis patients of European descent (Patsopoulos et al. 2011). Similarly, Tsoi et al. 2012 also conducted a meta-analysis study on the immune-mediated inflammatory disease of the skin, psoriasis (10,588 cases and 22,806 controls), and found TAGAP to be a disease susceptibility locus. Recently, Kumar et al. observed significant association between a bloodstream infection called candidaemia and SNPs in TAGAP in patients of European ancestry (Kumar et al. 2014). A population-based study on North Indian population via replication analysis has also shown a significant association of TAGAP polymorphism with RA (Prasad et al. 2012). A similar study by Perkins et al. reported potential susceptibility of SNPs in TAGAP with RA in African Americans (Perkins et al. 2012). In another study, Chen et al. 2011 conducted a comprehensive imputation of CEU HapMap single-nucleotide polymorphisms in a GWA study of 5500 RA cases and 22621 controls (all of European ancestry) to fine map the TAGAP risk locus in RA and the strongest signal of association was to an imputed SNP, rs212389. Although our results are conflicting with this particular study, these differences could be due to dissimilarity in origin and ethnicity of both populations. Fine mapping of TAGAP have 
been performed in European populations (Chen et al. 2011), similar studies in Pakistani population might yield additional insights.

\subsection{Conclusion}

Given that TAGAP is involved in activation of T-cells, variations in this gene might be important in the pathogenesis of autoimmune disorders. This is the first TAGAP association study with RA in Pakistani cohort. The results support the hypothesis of risk genes in complex disorders being population specific. Further genetics association studies in larger group of patients from various ethnicities are required so that the risk polymorphisms in TAGAP can be fine mapped. Moreover, future functional studies are considered necessary to further explore the significance of these polymorphisms which will be helpful in investigating their biological effects and any potential role in RA pathogenesis that might lead to the identification of novel therapeutic targets for RA.

\section{Acknowledgments}

We are thankful to all healthy volunteers, patients and rheumatologists for their cooperation and participation in the study. Help provided by lab members of Kennedy Institute of Rheumatology, University of Oxford, is greatly acknowledged.

Funding: This work was supported by the Higher Education Commission of Pakistan [grant number: IRSIP 26 BMS 53]

\section{References}

Arnett, F.C., Edworthy, S.M., Bloch, D.A., McShane, D.J., Fries, J.F., Cooper, N.S., et al. 1988 The American Rheumatism Association 1987 revised criteria for the classification of Rheumatoid Arthritis. Arthritis. Rheum. 31, 315-324.

Chatzikyriakidou, A., Voulgari, P.V., Lambropoulos, A., Georgiou, I., Drosos, A.A., 2013. Validation of the TAGAP rs212389 polymorphism in rheumatoid arthritis susceptibility. Joint. Bone. Spine. 38, 541-555. 
Connelly, T.M., Sehgal, R., Berg, A.S., Hegarty, J.P., Deiling, S., Stewart, D.B., Poritz, L.S., Koltun, W.A., 2012. Mutation in TAGAP is protective of anal sepsis in ileocolic Crohn's disease. Dis. Colon. Rectum. 55, 1145-1152.

Connelly, T.M., Berg, A.S., Harris, L.R., Hegarty, J.P., Ruggiero, F.M., Deiling, S.M., Brinton, D.L., Koltun, W.A. 2014. T-cell activation Rho GTPase-activating protein expression varies with inflammation location and severity in Crohn's disease. J. Surg. Res. 190, 457-464.

Chen, R., Stahl, E.A., Kurreeman, F.A., Gregersen, P.K., Siminovitch, K.A., Worthington, J., Padyukov, L., Raychaudhuri, S., Plenge, R.M., 2011 Fine mapping the TAGAP risk locus in rheumatoid arthritis. Genes. Immun. 12, 314.

Chang, M., Rowland, C.M., Gracia, V.E., Schrodi, S.J., Catanese, J.J., van der Helm-van Mil, A.H., et al. 2008. A Large-Scale Rheumatoid Arthritis Genetic Study Identifies Association at Chromosome 9q33.2. PLoS. Genet. 4, e1000107.

Eyre, S., Hinks, A., Bowes, J., Flynn, E., Martin, P., Wilson, A.G., et al. 2010. Overlapping genetic susceptibility variants between three autoimmune disorders: rheumatoid arthritis, type 1 diabetes and celiac disease. Arthritis. Res. Ther. 12, R175.

Franke, A., McGovern, D., Barret, J., Wang, K., Radford-Smith, G.L., Ahmad, T., et al. 2010. Genome-wide metaanalysis increases to 71 the number of confirmed Crohn's disease susceptibility loci. Nat. Genet. 42, 1118.

Festen, E., Goyette, P., Green, T., Boucher, G., Beauchamp, C., Trynka, G., et al. 2011. A metaanalysis of genome-wide association scans identifies IL18RAP, PTPN2, TAGAP and PUS10 as Shared risk loci for Crohn's disease and celiac disease. PLoS. Genet. 7, e1001283.

Firestein, G.S., 2003 Evolving concepts of rheumatoid arthritis. Nature. 423, 356-361.

Farooqi, A., Gibson, T., 1998. Prevalence of the major rheumatic disorders in the adult population of North Pakistan. Br. J. Rheumatol. 37, 491-495.

Gibofsky, A., Winchester, R.J., Patarroyo, M., Fotino, M., Kunkel, H.G., 1978. Disease associations of the Ia-like human alloantigens: contrasting patterns in rheumatoid arthritis and systemic lupus erythematosus. J. Exp. Med. 148, 1728-1732.

Gonzalez-Gay, M.A., Garcia-Porrua, C., Hajeer, A.H., 2002. Influence of human leukocyte antigen-DRB1 on the susceptibility and severity of rheumatoid arthritis. Semin. Arthritis. Rheum. 31, 355-360. 
Gregersen, P.K., Silver, J., Winchester, R.J., 1987. The shared epitope hypothesis. An approach to understanding the molecular genetics of susceptibility to rheumatoid arthritis. Arthritis. Rheum. 30, 1205-1213.

Hameed, K., Gibson, T., Kadir, M., Sultana, S., Fatima, Z., Syed, A., 1995. The prevalence of rheumatoid arthritis in affluent and poor urban communities of Pakistan. Br. J. Rheumatol. $34,252-256$.

Hinks, A., Barton, A., John, S., Bruce, I., Hawkins, C., Griffiths, C.E., Donn, R., Thomson, W., Silman, A., Worthington, J., 2005. Association between the PTPN22 gene and rheumatoid arthritis and juvenile idiopathic arthritis in a UK population: further support that PTPN22 is an autoimmunity gene. Arthritis. Rheum. 52, 1694-1699.

Honne, K., Hallgrímsdóttir, I., Wu, C., Sebro, ～R., Jewell, N.P., Sakurai, T., Iwamoto, M., Minota, S., Jawaheer, D., 2016. A longitudinal genome-wide association study of antitumor necrosis factor response among Japanese patients with rheumatoid arthritis. Arthritis. Res. Ther. 18, 12.

Julià, A., Fernandez-Nebro, A., Blanco, F., Ortiz, A., Cañete, J.D., Maymó, J., et al. 2016. A genome-wide association study identifies a new locus associated with the response to antiTNF therapy in rheumatoid arthritis. Pharmacogenomics. J. 16, 147-150.

Jalil, S.F., Bhatti, A., Demirci, F.Y., Wang, X., Ahmed, I., Ahmed, M., Barmada, M.M., Malik, J.M., John, P., Kamboh, M.I., 2013. Replication of European Rheumatoid Arthritis loci in a Pakistani Population. J. Rheumatol. 40, 401-407.

Kumar, V., Cheng, S.C., Johnson, M.D., Smeekens, S.P., Wojtowicz, A., GiamarellosBourboulis, E., et al. 2014. Immunochip SNP array identifies novel genetic variants conferring susceptibility to candidaemia. Nat. Commun. 5, 4675.

Kobayashi, S., Ikari, K., Kaneko, H., Kochi, Y., Yamamoto, K., Shimane, K., et al. 2008. Association of STAT4 with susceptibility to rheumatoid arthritis and systemic lupus erythematosus in the Japanese population. Arthritis. Rheum. 58, 1940-1946.

Kochi, Y., Okada, Y., Suzuki, A., Ikari, K., Terao, C., Takahashi, A., et al. 2010. A regulatory variant in CCR6 is associated with rheumatoid arthritis susceptibility. Nat. Genet. 42, 515-519. 
Lei, C., Dongqing, Z., Yeqing, S., Oaks, M.K., Lishan, C., Jianzhong, J., et al. 2005. Association of the CTLA-4 gene with rheumatoid arthritis in Chinese Han population. Eur. J. Hum. Genet. 13, 823-828.

Liu, M.F., Kohsaka, H., Sakurai, H., Azuma, M., Okumura, K., Saito, I., Miyasaka, N., 1996. The presence of costimulatory molecules CD86 and CD28 in Rheumatoid Arthritis synovium. Arthritis. Rheum. 39, 110-114.

McInnes, I.B., Schett, G., 2011. The pathogenesis of rheumatoid arthritis. N. Engl. J. Med. 365, 2205-2219.

Moreland, L., 2005. Unmet needs in rheumatoid arthritis. Arthritis. Res. Ther. 7, S2-S8.

Moreno, I., Valenzuela, A., García, A., Yélamos, J., Sánchez, B., Hernánz, W., 1996. Association of the shared epitope with radiological severity of rheumatoid arthritis. J. Rheumatol. 23, 6-9.

Mao, M., Biery, M.C., Kobayashi, S.V., Ward, T., Schimmack, G., Burchard, J., Schelter, J.M., Dai, H., He, Y.D., Linsley, P.S. 2004. T lymphocyte activation gene identification by coregulated expression on DNA microarray. Genomics. 83, 989-999.

Prasad, P., Kumar, A., Gupta, R., Juyal, R.C., Thelma, B.K., 2012 Caucasian and Asian Specific Rheumatoid Arthritis Risk Loci Reveal Limited Replication and Apparent Allelic Heterogeneity in North Indians. PLoS. ONE. 7, e31584.

Perkins, E.A., Landis, D., Causey, Z.L., Edberg, Y., Reynolds, R.J., Hughes, L.B., et al. 2012. Association of Single Nucleotide Polymorphisms (SNPs) in CCR6, TAGAP and TNFAIP3 with Rheumatoid Arthritis in African Americans. Arthritis. Rheum. 64, 1355-1358.

Patsopoulos, N.A., Esposito, F., Reischl, J., Lehr, S., Bauer, D., Heubach, J., et al. 2011. Genomewide meta-analysis identifies novel multiple sclerosis susceptibility loci. Ann. Neurol. 70, 897-912.

Raychaudhuri, S., Thomson, B.P., Remmers, E.F., Eyre, S., Hinks, A., Guiducci, C., et al. 2009. Genetic variants at CD28, PRDM1 and CD2/CD58 are associated with rheumatoid arthritis risk. Nat. Genet. 41, 1313-1318.

Stahl, E.A., Raychaudhuri, S., Remmers, E.F., Xie, G., Eyre, S., Thomson, B.P., et al. 2010. Genome-wide association study meta-analysis identifies seven new rheumatoid arthritis risk loci. Nat. Genet. 42, 508-514. 
Turesson, C., Jacobsson, L., Bergström, U., 1999. Extra-articular rheumatoid arthritis: prevalence and mortality. Rheumatology. 38, 668-674.

Turesson, C., Schaid, D.J., Weyand, C.M., Jacobsson, L.T., Goronzy, J.J., Petersson, I.F., et al. 2005. The impact of $H L A-D R B 1$ genes on extra-articular disease manifestations in rheumatoid arthritis. Arthritis. Res. Ther. 7, R1386-1393.

Tsoi, L.C., Spain, S.L., Knight, J., Ellinghaus, E., Stuart, P.E., Capon, F., et al. 2012. Identification of fifteen new psoriasis susceptibility loci highlights the role of innate immunity. Nat. Genet. 44, 1341-1348. 


\section{Table 1}

\section{Characteristics of RA patients and controls}

\begin{tabular}{|lcc|}
\hline Characteristics & Cases (n=186) & Controls (n=185) \\
\hline $\begin{array}{l}\text { Rheumatoid factor } \\
\text { positiveness (\%) }\end{array}$ & 95 & 0 \\
$\begin{array}{l}\text { Anti-cyclic citrullinated } \\
\text { peptide positiveness (\%) }\end{array}$ & 92 & 0 \\
Female (\%) & 76 & 36 \\
Mean Age, Years $( \pm \mathrm{SD})^{*}$ & $43 \pm 12$ & $40 \pm 11$ \\
Disease Duration, Years $( \pm \mathrm{SD})$ & $6.5 \pm 5$ & 0 \\
\hline
\end{tabular}

Where, $n=$ number of subjects

* standard deviation 
Table 2

Genotype frequencies distribution in cases and controls ( $\chi^{2}$ test)

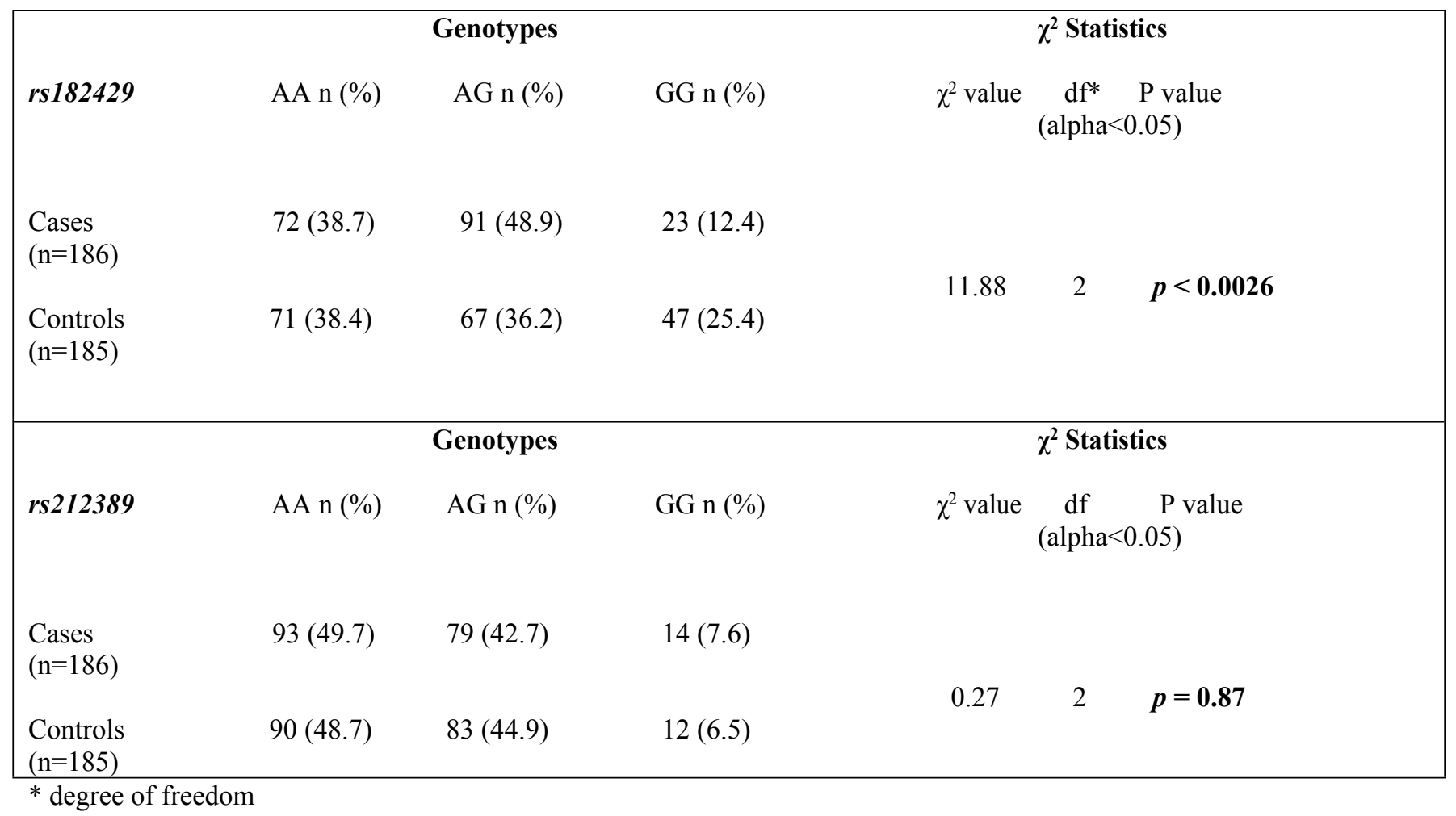


Table 3

Allele frequencies distribution in cases and controls

\begin{tabular}{|c|c|c|c|}
\hline & \multicolumn{2}{|c|}{ Alleles } & $A$ allele vs $G$ allele \\
\hline rs182429 & A n $(\%)$ & G n $(\%)$ & $\begin{array}{c}\mathrm{OR}^{*}\left(95 \% \mathrm{CI}^{* *}\right) \\
P \text { value }\end{array}$ \\
\hline $\begin{array}{l}\text { Cases } \\
(\mathrm{n}=186)\end{array}$ & $235(63.2)$ & $137(36.8)$ & $\begin{array}{l}1.32(0.98-1.77) \\
p=\mathbf{0 . 0 3 7 3}\end{array}$ \\
\hline \multirow[t]{2}{*}{$\begin{array}{l}\text { Controls } \\
(n=185)\end{array}$} & $209(56.5)$ & $161(43.5)$ & \\
\hline & \multicolumn{2}{|c|}{ Alleles } & A allele vs $G$ allele \\
\hline$r s 212389$ & $\operatorname{An}(\%)$ & G n $(\%)$ & $\begin{array}{c}\mathrm{OR}^{*}\left(95 \% \mathrm{CI}^{* *}\right) \\
P \text { value }\end{array}$ \\
\hline $\begin{array}{l}\text { Cases } \\
(n=186)\end{array}$ & $265(71.2)$ & $107(28.8)$ & $1.00(0.73-1.37)$ \\
\hline $\begin{array}{l}\text { Controls } \\
(n=185)\end{array}$ & $263(71.2)$ & $107(28.8)$ & $p=1.00$ \\
\hline
\end{tabular}


Table 4

Genotype and allele frequencies in cases and controls (Z-test)

\begin{tabular}{|c|c|c|c|c|c|c|}
\hline \multirow[b]{2}{*}{$r s 182429$} & \multicolumn{3}{|c|}{ Genotypes } & \multicolumn{2}{|c|}{ Alleles } & \multirow{4}{*}{$\begin{array}{l}Z \text {-Value }=1.69 \\
P \text {-Value }<0.091\end{array}$} \\
\hline & AA n $(\%)$ & AG n $(\%)$ & GG n $(\%)$ & $\mathrm{A}(\%)$ & $\mathrm{G}(\%)$ & \\
\hline $\begin{array}{l}\text { Cases } \\
(\mathrm{n}=186)\end{array}$ & $72(38.7)$ & $91(48.9)$ & $23(12.4)$ & 0.45 & 0.55 & \\
\hline \multirow[t]{2}{*}{$\begin{array}{l}\text { Controls } \\
(\mathrm{n}=185)\end{array}$} & $71(38.4)$ & $67(36.2)$ & $47(25.4)$ & 0.51 & 0.49 & \\
\hline & \multicolumn{3}{|c|}{ Genotypes } & \multicolumn{2}{|c|}{ Alleles } & \\
\hline$r s 212389$ & AA n $(\%)$ & AG n $(\%)$ & GG n $(\%)$ & $\mathrm{A}(\%)$ & $\mathrm{G}(\%)$ & \\
\hline $\begin{array}{l}\text { Cases } \\
(\mathrm{n}=186)\end{array}$ & $93(49.7)$ & $79(42.7)$ & $14(7.6)$ & 0.71 & 0.29 & $Z-$ Value $=0.00$ \\
\hline $\begin{array}{l}\text { Controls } \\
(\mathrm{n}=185)\end{array}$ & $90(48.7)$ & $83(44.9)$ & $12(6.5)$ & 0.71 & 0.29 & $P$-Value $=1.00$ \\
\hline
\end{tabular}



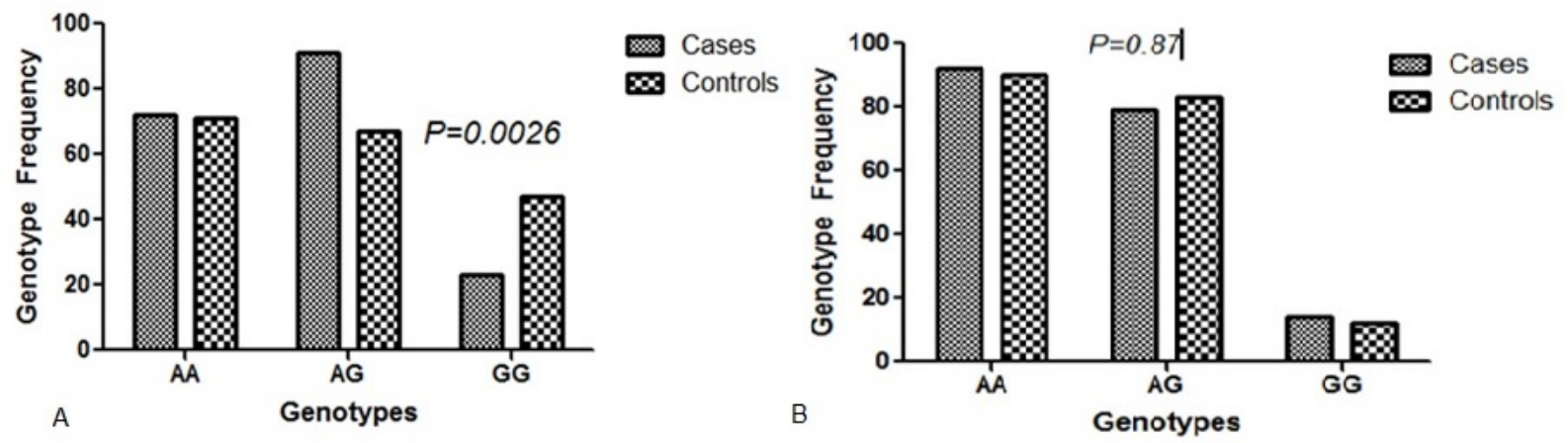

Fig.1. Genotype frequencies in RA cases and healthy controls: (A) Genotype frequency for $r s 182429$ showing significantly higher heterozygous AG genotype in cases than in controls with $\mathrm{P}=0.0026$. (B) Genotype frequency in cases and controls for $r s 212389$ showing no significant distribution with $\mathrm{P}=0.87$
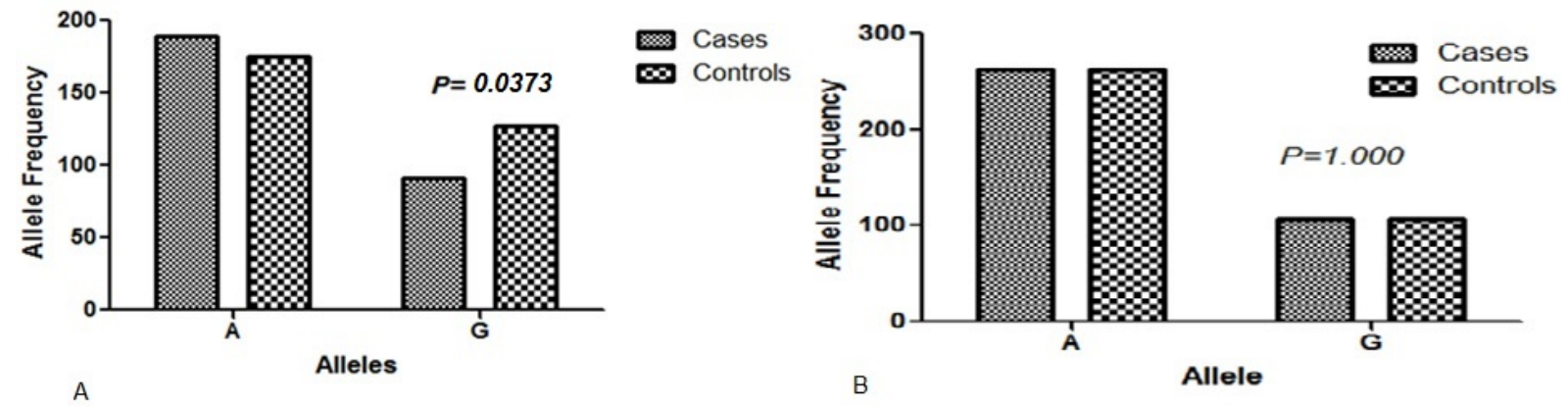

Fig.2. Allele frequency in RA cases and healthy controls: (A) Allelic frequency for $r s 182429$ showing higher frequency of allele $\mathrm{A}$ in cases than in controls with $\mathrm{P}=0.0373$. (B) Allele frequency for $r s 212389$ in Cases and Controls with $\mathrm{P}=1.0$. 